\title{
Symbolic Test Selection Based on Approximate Analysis ${ }^{\star}$
}

\author{
Bertrand Jeannet, Thierry Jéron, Vlad Rusu, and Elena Zinovieva \\ IRISA/INRIA, Campus de Beaulieu, Rennes, France \\ \{bjeannet, jeron, rusu, lenaz\}@irisa.fr
}

\begin{abstract}
This paper addresses the problem of generating symbolic test cases for testing the conformance of a black-box implementation with respect to a specification, in the context of reactive systems. The challenge we consider is the selection of test cases according to a test purpose, which is here a set of scenarios of interest that one wants to observe during test execution. Because of the interactions that occur between the test case and the implementation, test execution can be seen as a game involving two players, in which the test case attempts to satisfy the test purpose.

Efficient solutions to this problem have been proposed in the context of finite-state models, based on the use of fixpoint computations. We extend them in the context of infinite-state symbolic models, by showing how approximate fixpoint computations can be used in a conservative way. The second contribution we provide is the formalization of a quality criterium for test cases, and a result relating the quality of a generated test case to the approximations used in the selection algorithm.
\end{abstract}

\section{Introduction}

In this paper we address the generation of test cases in the framework of conformance testing of reactive systems [1. In this context, a Test Case $(T C)$ is a program run in parallel with a black-box Implementation Under Test (IUT), that stimulates the IUT by repeatedly sending inputs and checking that the observed outputs of the $I U T$ are in conformance with a given specification $S$. In case the IUT exhibits a conformance error, the execution is immediately interrupted. However, in addition to checking the conformance of the $I U T$, the goal of the test case is to guide the parallel execution towards the satisfaction of a test purpose, typically a set of scenarios of interest. Because of this second feature, test execution can be seen as a game between two programs, the test case and the IUT. The test case wins if it succeeds to make the parallel execution realize one of the interesting scenarios specified by the test purpose; the IUT wins if the execution cannot be extended any more to one that realizes an interesting scenario. If a conformance error is detected, the game terminates with a tie.

The test selection problem consists in finding a strategy that minimizes the likehood for the test case to lose the game. Indeed, it is generally not possible

\footnotetext{
* The full version of this paper is available as Irisa report 11 .
} 
to ensure that the test case wins, because IUT is unknown: it is a black-box program that may behave in a non-controllable way. This problem has been previously addressed in a context where the specifications, the test cases and the test purposes are modeled with finite Labelled Transition Systems (LTS) 9].

Finding a suitable strategy for the test case is decomposed in two steps:

1. One first performs an off-line selection of a Test Case that detects when the game is lost by the tester and stops the execution in this case. This is done by static analysis of the specification $S$ and the test purpose TP.

2. Then, during the execution of the obtained test case in parallel with the $I U T$, one performs an on-line selection of the inputs that the test case sends to the IUT. This on-line selection is based on the history of the current execution.

A previous paper 14 extends these principles and algorithmic methods to the case where specifications, test purposes and test cases are modeled with Input-Output Symbolic Transition Systems (ioSTS), which are automata that operate on variables (integers, booleans, aggregate types, ...) and communicate with the environment by means of input and output actions carrying parameters. For undecidability reason, the static analysis used for the off-line selection (Step 1) is approximated. [14 considers only a specific analysis (moreover restricted to the control structure) and does not study the effect of the approximations on the generated test cases.

The contributions of this paper are twofold. First we describe a general test selection method parameterized by an approximate analysis, in the context of Input-Output Symbolic Transition Systems. Compared to [14], we allow for the use of more precise analyses that perform both control and data based selection. We show that the test cases obtained by this method are sound. Second, we investigate the effect of the approximations of the analysis from the point of view of test execution as a game: in which way do they degrade the winning capabilities of the obtained test case? This leads us to define an accuracy ordering between test cases, to formalize the notion of optimal test case, and to compare the test cases generated by our method using these notions.

\section{Context and Related Work}

Conformance testing: Testing is the most used validation technique to assess the quality of software systems. Among the aspects of software that can be tested, e.g., functionality, performance, timing, robustness, etc, we focus here on conformance testing and specialize it to reactive systems. In this approach, the software is specified in a behavioral model which serves both as a basis for test generation and for verdicts assignment. Testing theories based on models such as automata associated to fault models (see e.g. the survey [13]), or labelled transition systems with conformance relations (see e.g. [16]) are now well understood. Test generation algorithms have been designed based on these theories, and tools like TorX [2], TGV [9] have been developed and used on industrial-size systems.

Test selection: The test selection problem consits of choosing some test cases among many possible, according to a given criterion. Most approaches are based 
on variants of classical control and data-flow coverage criteria [8, 3, while others focus on specific functionalities using test purposes [6. Although this is not always made explicit, test generation typically relies on reachability and coreachability analyses [9] based on pre- and post- predicate transformers.

Symbolic models: Many of the existing test generation algorithms and tools operate on variants of labeled transition systems (LTS). High-level specifications (written in languages such as SDL, Lotos, or UML) can be treated as well, via a translation (by state-space exploration) to the more basic labeled transition systems. More recently, attempts have been made in the direction of symbolic test generation [14] which works directly on the higher-level specifications without enumerating their state-space, thus avoiding the state-space explosion problem.

Outline: In Section 2 we recall ioLTS model, the corresponding testing theory and the principles of test generation using test purposes. In Section 3, we define the syntax of the symbolic model of ioSTS and its ioLTS semantics. In Section 4 we propose an off-line test selection algorithm for ioSTS based on syntactical transformations and parameterized by an approximate fixpoint analysis. Section [5] describes the on-line test selection that occurs during test execution. Section [6 defines qualitative properties on tests cases concerning their ability to satisfy the test purpose, and shows how the approximations used in the off-line test generation step influence those qualities.

\section{Testing with Input/Output Labeled Transition Systems}

Specification languages for reactive systems can often be given a semantics in terms of labelled transition systems. For test generation, we use the following version where actions are explictly partitioned into inputs, which are controlled by the environment, and outputs, which the environment may only observe. This model also serves as a semantic model for our symbolic automata (cf. Section 3).

Definition 1 (ioLTS). An Input/Output Labelled Transition System is a tuple $\left(Q, Q_{0}, \Lambda, \rightarrow\right)$ where $Q$ is a set of states, $Q_{0}$ the set of initial states, $\Lambda=\Lambda_{?} \cup \Lambda_{!}$is a set of actions partitioned into inputs ( $\Lambda_{\text {? }}$ ) and outputs ( $\Lambda_{!}$) and $\rightarrow \subseteq Q \times \Lambda \times Q$ is the transition relation.

We write $q \stackrel{\alpha}{\rightarrow} q^{\prime}$ in place of $\left(q, \alpha, q^{\prime}\right) \in \rightarrow$ and note $q \stackrel{\alpha}{\rightarrow}$ when $\exists q^{\prime}: q \stackrel{\alpha}{\rightarrow} q^{\prime}$.

For the sake of simplicity, we consider only deterministic ioLTS: the alphabet does not contain internal actions and $\forall q \in Q, q \stackrel{\alpha}{\rightarrow} q^{\prime} \wedge q \stackrel{\alpha}{\rightarrow} q^{\prime \prime} \Rightarrow q^{\prime}=q^{\prime \prime}$.

A run is a finite sequence $\rho=q_{0} \stackrel{\alpha_{0}}{\rightarrow} q_{1} \stackrel{\alpha_{1}}{\rightarrow} \ldots \stackrel{\alpha_{n-1}}{\rightarrow} q_{n}$ such that $q_{0} \in$ $Q_{0}$ and $\forall i<n,\left(q_{i}, \alpha_{i}, q_{i+1}\right) \in \rightarrow$. Its projection onto actions is the trace $\sigma=$ $\operatorname{trace}(\rho)=\alpha_{0} \ldots \alpha_{n-1}$. We denote by Runs $(M) \subseteq Q_{0} \cdot(\Lambda \cdot Q)^{*}$ the set of runs of $M$ and by $\operatorname{Traces}(M) \subseteq \Lambda^{*}$ the set of traces of $M$. An ioLTS $M$ can be seen as an automaton if it is equipped with a set of marked states $X \subseteq Q$. A run $\rho=q_{0} \stackrel{\alpha_{0}}{\rightarrow} q_{1} \stackrel{\alpha_{1}}{\rightarrow} \ldots \stackrel{\alpha_{n-1}}{\rightarrow} q_{n}$ is accepted in $X$ iff $q_{n} \in X$. We denote Runs $_{X}(M) \subseteq$ Runs $(M)$ the set of runs accepted by $X$. Similarly, the set of 
accepted traces $\operatorname{Traces}_{X}(M) \subseteq \operatorname{Traces}(M)$ is obtained by projecting $\operatorname{Runs}_{X}(M)$ on $\Lambda^{*}$. An ioLTS $M$ is complete in a state $q$ if $\forall \alpha \in \Lambda: q \stackrel{\alpha}{\rightarrow}$; it is complete if it is complete in all states. Similarly, the notion of input-completeness is defined by replacing $\Lambda$ with $\Lambda_{\text {? }}$.

The synchronous product of two ioLTS $M=\left(Q, Q_{0}, \Lambda, \rightarrow^{M}\right)$ and $M^{\prime}=$ $\left(Q^{\prime}, Q_{0}^{\prime}, \Lambda, \rightarrow^{M^{\prime}}\right)$ with same alphabet is the ioLTS $M \times M^{\prime}=\left(Q \times Q^{\prime}, Q_{0} \times\right.$ $Q_{0}^{\prime}, \Lambda, \rightarrow^{M \times M^{\prime}}$ ) where $\rightarrow^{M \times M^{\prime}}$ is defined by the inference rule:

$$
\frac{q_{1} \stackrel{\alpha}{\rightarrow}{ }^{M} q_{2} \quad q_{1}^{\prime} \stackrel{\alpha}{\rightarrow}{ }^{M^{\prime}} q_{2}^{\prime}}{\left(q_{1}, q_{1}^{\prime}\right) \stackrel{\alpha}{\rightarrow} \times M^{M^{\prime}}\left(q_{2}, q_{2}^{\prime}\right)}
$$

As usual, we get $\operatorname{Traces}\left(M \times M^{\prime}\right)=\operatorname{Traces}(M) \cap \operatorname{Traces}\left(M^{\prime}\right)$ and Traces $_{X \times X^{\prime}}\left(M \times M^{\prime}\right)=$ Traces $_{X}(M) \cap$ Traces $_{X^{\prime}}\left(M^{\prime}\right)$ for $X \subset Q$ and $X^{\prime} \subset Q^{\prime}$.

For a set of traces $W \subseteq \Lambda^{*}$, we denote respectively by $\operatorname{pref}_{\leq}(W)$ and pref $<(W)$ the set of prefixes (resp. strict prefixes) of $W$. For $X \subseteq Q$, we denote by $\operatorname{post}(X)=\left\{q^{\prime} \mid \exists q \in X, \exists \alpha \in \Lambda: q \stackrel{\alpha}{\rightarrow} q^{\prime}\right\}$ and $\operatorname{pre}(X)=\left\{q \mid \exists q^{\prime} \in X, \exists \alpha \in \Lambda:\right.$ $\left.q \stackrel{\alpha}{\rightarrow} q^{\prime}\right\}$ the pre- and post-condition operators. The set of states reachable from a subset $Q^{\prime}$ of $Q$ in $M$ may then be defined by reach $\left(Q^{\prime}\right)=\operatorname{lfp}\left(\lambda X \cdot Q^{\prime} \cup \operatorname{post}(X)\right)$ where lfp is the least fixpoint operator. Similarly, the set of states coreachable from a set of states $Q^{\prime}$ may be defined as $\operatorname{coreach}\left(Q^{\prime}\right)=\operatorname{lfp}\left(\lambda X . Q^{\prime} \cup \operatorname{pre}(X)\right)$.

\section{$2.1 \quad$ Testing Theory}

The testing theory we consider is based on the notions of specification, implementation, and conformance relation between them [16. The specification is an ioLTS $S=\left(Q^{S}, Q_{0}^{S}, \Lambda, \rightarrow^{S}\right)$. The Implementation Under Test $(I U T)$ is also assumed to be an ioLTS IUT $=\left(Q^{I U T}, Q_{0}^{I U T}, \Lambda, \rightarrow^{I U T}\right)$ which is unknown except for its alphabet, which is assumed to be the same as that of the specification. Moreover, it is assumed that the IUT is input-complete, which reflects the hypothesis that the implementation cannot refuse an input from its environment.

Definition 2 (Conformance relation). $A$ trace $\sigma$ is conformant to $S$, denoted by $\sigma$ conf $S$, iff pref $\leq(\sigma) \cap\left(\operatorname{Traces}(S) \cdot \Lambda_{!}\right) \subseteq \operatorname{Traces}(S)$. IUT is conformant to $S$, denoted by IUT conf $S$, iff Traces $(I U T) \cap \operatorname{Traces}(S) \cdot \Lambda_{!} \subseteq \operatorname{Traces}(S)$.

Intuitively, IUT conf $S$ if after each trace of $S$, IUT may emit only outputs that $S$ can emit as well while its inputs are unconstrained. For readers familiar with ioco [16], note that conf can be interpreted as ioco if $S$ makes quiescence (absence of output) explicit. For instance on the figure $\neg\left(\sigma_{1}\right.$ conf $\left.S\right), \sigma_{2}$ conf $S$, and $\sigma_{0}$ conf $S$ as $\sigma_{0}$ diverges from $S$ by an input.

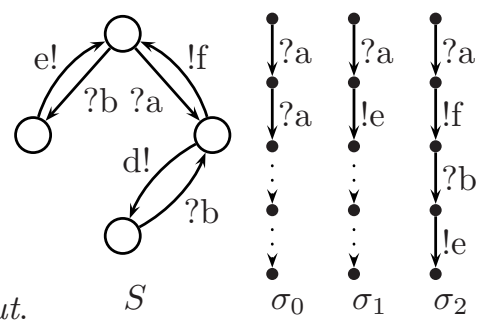

\subsection{Test Cases and Test Purposes}

A test case is an ioLTS able to interact with an implementation and to emit verdicts about the implementation's conformance with respect to the specification. 
Table 1. Properties of Test Cases

\begin{tabular}{|c|c|}
\hline Property & Explanations \\
\hline (1) $T C$ is & always, def 3 \\
\hline (2) $\operatorname{Traces}(T C) \subseteq \operatorname{Traces}(S) \cdot\left(\{\epsilon\} \cup \Lambda_{!}\right)$ & ef 3 \\
\hline (3) $\operatorname{Traces}_{\text {Fail }}(T C)=\operatorname{Traces}(T C) \cap((\operatorname{Trace}$ & , def 5 \\
\hline (4) Traces $_{\text {Pass }}(T C)=$ Traces $(T C) \cap A T r a$ & , def 5 \\
\hline (5) $\operatorname{Traces}_{\text {Inconc }}(T C) \subseteq R \operatorname{Traces}(S, T P)$ & $\operatorname{def} 5$ \\
\hline (6) $\operatorname{Traces}_{\text {Inconc }}(T C)=\operatorname{Trac}$ & optimality, def 1 \\
\hline $\begin{array}{r}\cap \cap \operatorname{pref}_{<}(\operatorname{ATraces}(S, T P)) \cdot \Lambda_{\text {! }} \\
\text { (7) } \operatorname{Traces}(T C) \cap \Lambda^{*} \cdot \Lambda_{\text {? }} \subseteq \operatorname{pref}_{<}(\operatorname{ATraces}(S, T P))\end{array}$ & optimality, de \\
\hline
\end{tabular}

In our approach, a test case is an ioLTS implementing a strategy for satisfying a given test purpose (typically, staying within a (finite or infinite) set of traces). The test case takes into account output choices of the specification (observable non-determinism) and anticipates incorrect outputs of the implementation.

Definition 3 (Test Case). A test case for a specification $S$ is an ioLTS $T C=\left(Q^{T C}, Q_{0}^{T C}, \Lambda^{T C}, \rightarrow^{T C}\right)$ equipped with 3 disjoint subsets of sink states Pass, Fail, Inconc $\subseteq Q^{T C}$ (corresponding to the verdicts it may emit) such that

- its alphabet is the mirror of that of $S\left(\Lambda_{?}^{T C}=\Lambda_{!}^{S}\right.$ and $\left.\Lambda_{!}^{T C}=\Lambda_{?}^{S}\right)$ and it is input-complete (outputs of IUT are not refused) except in verdict states;

- Traces $(T C) \subseteq \operatorname{Traces}(S) \cdot\left(\{\epsilon\} \cup \Lambda_{!}\right)$: as soon as a conformance error cannot occur any more the test case stops (cf. Definition Q2).

The specification $S$ contains all the information relevant to conformance, and its mirror followed by input-completion constitutes a test case by itself. However, such a test case is typically too large and is not focused on any part of the system. It is more interesting in practice to test what happens in the course of a given scenario (or set thereof), and if no error has been detected, to end the test successfully when the scenario is completed. This is precisely the reason for introducing test purposes.

Definition 4 (Test Purpose). A test purpose TP for a specification $S$ is an $i o L T S T P=\left(Q^{T P}, Q_{0}^{T P}, \Lambda, \rightarrow^{T P}\right)$ equipped with a subset Accept $\subseteq Q^{T P}$ of accepting states, which are sink. TP is complete except in Accept states. TP defines a set ATraces $(S, T P)$ of accepted traces of $S$ which induces a set RTraces $(S, T P)$ of refused traces (traces of $S$ that cannot be extended to accepted traces):

$$
\begin{aligned}
& \operatorname{ATraces}(S, T P)=\operatorname{Traces}_{Q^{S} \times \operatorname{Accept}}(S \times T P) \\
& R \operatorname{Traces}(S, T P)=\operatorname{Traces}(S) \backslash \operatorname{pref}_{\leq}(\operatorname{ATraces}(S, T P))
\end{aligned}
$$

Observe that both accepted and refused traces are conformant. More elaborate test purposes can be defined which may choose traces based on internal actions and states. We do not describe them here for simplicity.

A test case $T C$ should emit the appropriate verdicts in the appropriate situations. Fail verdicts should be emitted if and only if a non conformance is observed. This requirement depends only on $S$. Additionally, since a test purpose $T P$ is 
used for selection, $T C$ and $I U T$ can be viewed as players in a game. In this context, Pass verdicts should reflect success of the game for the test case, while Inconc verdicts should reflect defeat for the test case. These requirements are made explicit in the following definition:

Definition 5 (Soundness of test case verdicts). The verdicts of $T C$ are sound w.r.t. $S$ and TP whenever the following properties of Table 1 are satisfied:

- (3): Fail is emitted iff TC observes an unspecified output after a trace of $S$.

- (4): Pass is emitted iff TC observes a trace of $S$ accepted by TP.

- (5): Inconc may be emitted only if the trace observed by TC belongs to $S$ (thus, it is conformant) but is refused by TP. In this case, the test execution can be interrupted, as Pass cannot be emitted any more.

Notice that for test cases satisfying Definition 5, Fail and Pass verdicts are uniquely defined, so that they are emitted appropriately and as soon as possible. In particular for Fail, the requirement is stronger than the usual notion of soundness [16] which says that only non conformant IUTs can be rejected. On the other hand, Definition [5 does not uniquely define the Inconc verdict. We have adopted this definition in anticipation of the general (symbolic) test selection algorithm (addressed in Section 4) where checking whether a trace is refused is undecidable.

\subsection{Off-line Test Selection Algorithm}

For finite ioLTS, the principles of test generation using test purposes [12] are described by Table 2, Explanations and sketch of proof are given below.

1. After Step 1, by properties of $\times$ Traces $_{\text {Pass }}(P)=\operatorname{ATraces}(S, T P)$, implying Property (4) of Table 1, and Traces $(P) \subseteq \operatorname{Traces}(S)$, implying Property (2). Intuitively, the product $P$ combines information about conformance, coming from $S$, and information about the game with the IUT, coming from TP.

2. After Step 2, we have $\operatorname{Traces}_{\text {Inconc }}\left(P^{\prime}\right) \subseteq \operatorname{RTraces}(S, T P)$, implying Property (5) of Table 1 Properties (2) and (4) from the previous step are preserved. This selection step is based on the definition of $\operatorname{RTraces}(S, T P)$ and the following property: $\operatorname{pref}_{\leq}\left(\operatorname{Traces}_{\text {Pass }}(P)\right)=\operatorname{Traces}_{\text {coreach }}($ Pass $(P)$. The exact knowledge of coreach (Pass) allows to detect when an action extends a trace and causes it to be refused (i.e., not a prefix any more of accepted traces).

3. After Step 3, we have $\operatorname{Traces}_{\text {Fail }}(T C)=\operatorname{Traces}(T C) \cap\left(\operatorname{Traces}(S) \cdot \Lambda_{!} \backslash\right.$ Traces $(S)$ ), which is Property (3) of Table 1, Moreover, Property (1) becomes true (rule (Fail $)$ ). Properties (2), (4), (5) are preserved by the transformation.

The TGV tool [9] is based on the above algorithm. The main optimization, consists in performing these operations on the fly. This means that $S, P, P^{\prime}$, and $T C$ are built in a lazy way, from a high level specification, thus avoiding the state explosion problem. This involves both a reachability and coreachability 
Table 2. Off-line test selection algorithm

\{ 1. Product and Pass verdict $\}$

$P:=S \times T P ;$ Pass $:=Q^{S} \times$ Accept $^{T P} ;$

\{2. Selection and Inconc verdict \}

$P^{\prime}=\left(Q^{P}, Q_{0}^{P}, \Lambda, \rightarrow^{P^{\prime}}\right)$ is equipped with Inconc $\subseteq Q^{P}$ and $\rightarrow^{P^{\prime}}$ is defined by:

$$
\begin{aligned}
& q, q^{\prime} \in \operatorname{coreach}\left(\text { Pass) } \quad q \in \operatorname { c o r e a c h } \left(\text { Pass) }, q^{\prime} \notin \operatorname{coreach}(\text { Pass) }\right.\right. \\
& \frac{q \stackrel{\alpha}{\rightarrow} P q^{\prime} \quad \alpha \in \Lambda_{?} \cup \Lambda_{!}}{q \stackrel{\alpha}{\rightarrow} P^{\prime} q^{\prime}} \quad\left(\text { KeepI) } \quad \frac{q \stackrel{\alpha}{\rightarrow} P q^{\prime} \quad \alpha \in \Lambda_{!}}{q \stackrel{\alpha}{\rightarrow} P^{\prime} q^{\prime} \quad q^{\prime} \in \text { Inconc }} \quad\right. \text { (Inconc) }
\end{aligned}
$$

\{ 3. Input-completion and Fail verdict \}

$$
\begin{aligned}
& T C=\left(Q^{P^{\prime}} \cup\{\text { Fail }\}, Q_{0}^{P}, \Lambda^{T C}, \rightarrow^{T C}\right) \text { with } \Lambda_{?}^{T C}=\Lambda_{!} \text {and } \Lambda_{!}^{T C}=\Lambda_{\text {? }} \text {, and } \\
& \rightarrow^{T C} \text { is defined by: }
\end{aligned}
$$

$$
\frac{q \stackrel{\alpha}{\longrightarrow} P^{\prime} q^{\prime}}{q \stackrel{\alpha}{\rightarrow} T C} q^{\prime} \quad(\mathrm{KeepF}) \quad \frac{\neg\left(q \stackrel{\alpha}{\rightarrow} P^{\prime}\right) \quad \alpha \in \Lambda !}{q \stackrel{\alpha}{\rightarrow} T C \text { Fail } \quad\left(\alpha \in \Lambda_{?}^{T C}\right)}
$$

analysis of $P$ that do not modify the soundness of the test case. A test case generated as above is called a complete test graph as it contains all traces accepted by TP. This notion will be formalized in Section 6. TGV also allows to generate other test cases that are less complete, by pruning some outputs and the corresponding subgraphs.

We do not describe the on-line selection phase, that occurs during the parallel execution of the test case with the IUT. It is described later for ioSTS.

\section{3 ioSTS: Input/Output Symbolic Transition Systems}

In this section, we introduce a model of symbolic automata with a finite set of locations and typed variables, which communicates with its environment through actions carrying values. We call it ioSTS for Input/Output Symbolic Transition Systems. Figure 1 gives an example of such an ioSTS.

Variables, Predicates, Assignments. In the sequel we shall assume a set of typed variables. We note $\mathcal{D}_{v}$ the domain in which a variable $v$ takes its values. For a set of variables $V=\left\{v_{1}, \ldots, v_{n}\right\}$, we note $\mathcal{D}_{V}$ the product domain $\mathcal{D}_{v_{1}} \times$ $\ldots \times \mathcal{D}_{v_{n}}$. An element of $\mathcal{D}_{V}$ is thus a vector of values for the variables in $V$. We use also the notation $\mathcal{D}_{\boldsymbol{v}}$ for a vector $\boldsymbol{v}$ of variables. Depending on the context, a predicate $P(V)$ on a set of variables $V$ may be considered either as a set $P \subseteq \mathcal{D}_{V}$, or as a logical formula, the semantics of which is a function $\mathcal{D}_{V} \rightarrow\{$ true, false $\}$. An assignment for a variable $v$ depending on the set of variables $V$ is a function of type $\mathcal{D}_{V} \rightarrow \mathcal{D}_{v}$. An assignment for a set $X$ of variables is then a function of type $\mathcal{D}_{V} \rightarrow \mathcal{D}_{X}$. We do not specify the syntactical constructions used for building predicates and assignments. They are discussed in the full paper.

Definition 6 (ioSTS). An Input/Output Symbolic Transition System $\mathcal{M}$ is defined by a tuple $(V, \Theta, \Sigma, T)$ where: 
$-V=V_{p} \cup V_{o}$ is the set of variables, partitioned into a set $V_{p}$ of proper variables and a set $V_{o}$ of observed variables.

- $\Theta$ is the initial condition: a predicate $\Theta \subseteq \mathcal{D}_{V_{p}}$ defined on proper variables.

$-\Sigma=\Sigma_{?} \cup \Sigma_{!}$is the finite alphabet of actions. Each action a has a signature $\operatorname{sig}(a)$, which is a tuple of types $\operatorname{sig}(a)=\left\langle t_{1}, \ldots, t_{k}\right\rangle$ specifying the types of the communication parameters carried by the action.

- $T$ is a finite set of symbolic transitions. A symbolic transition $t=$ $(a, \boldsymbol{p}, G, A)$, also noted $\left[a(\boldsymbol{p}): G(\boldsymbol{v}, \boldsymbol{p}) ? \boldsymbol{v}_{p}^{\prime}:=A(\boldsymbol{v}, \boldsymbol{p})\right]$, is defined by $(i)$ an action $a \in \Sigma$ and a tuple of (formal) communication parameters $\boldsymbol{p}=\left\langle p_{1}, \ldots, p_{k}\right\rangle$, which are local to a transition; without loss of generality, we assume that each action a always carries the same vector $\boldsymbol{p}$, which is supposed to be well-typed w.r.t. the signature $\operatorname{sig}(a)=\left\langle t_{1}, \ldots, t_{k}\right\rangle ; \mathcal{D}_{p}$ is denoted by $\mathcal{D}_{\operatorname{sig}(a)} ;($ ii $)$ a guard $G \subseteq \mathcal{D}_{V} \times \mathcal{D}_{\operatorname{sig}(a)}$, which is a predicate on the variables and the communication parameters, and (iii) an assignment $A: \mathcal{D}_{V} \times \mathcal{D}_{\operatorname{sig}(a)} \rightarrow \mathcal{D}_{V_{p}}$, which defines the evolution of the proper variables. We denote by $A_{v}$ the function in $A$ defining the evolution of the variable $v \in V_{p}$.

This model is rather standard, except for the distinction between proper and observed variables. The observed variables allow an observer ioSTS $\mathcal{M}_{1}$ to inspect the variables of another ioSTS $\mathcal{M}_{2}$ when composed together with it. Note also that there is no explicit notion of control location, since the control structure of an automaton can be encoded by a specific program counter variable.

The semantics of an ioSTS $\mathcal{M}=(V, \Theta, \Sigma, T)$ is an ioLTS $\llbracket \mathcal{M} \rrbracket=\left(Q, Q_{0}, \Lambda, \rightarrow\right)$ :

$-Q=\mathcal{D}_{V}, Q_{0}=\left\{\boldsymbol{\nu}=\left\langle\boldsymbol{\nu}_{p}, \boldsymbol{\nu}_{o}\right\rangle \mid \boldsymbol{\nu}_{p} \in \Theta \wedge \boldsymbol{\nu}_{o} \in \mathcal{D}_{V_{o}}\right\}$

$-\Lambda=\left\{\langle a, \boldsymbol{\pi}\rangle \mid a \in \Sigma \wedge \boldsymbol{\pi} \in \mathcal{D}_{\operatorname{sig}(a)}\right\}$

$\rightarrow$ is defined by

$$
\begin{gathered}
(a, \boldsymbol{p}, G, A) \in T \quad \boldsymbol{\nu}=\left\langle\boldsymbol{\nu}_{p}, \boldsymbol{\nu}_{o}\right\rangle \in \mathcal{D}_{V} \quad \boldsymbol{\pi} \in \mathcal{D}_{\operatorname{sig}(a)} \quad G(\boldsymbol{\nu}, \boldsymbol{\pi}) \\
\boldsymbol{\nu}^{\prime}=\left\langle\boldsymbol{\nu}_{p}^{\prime}, \boldsymbol{\nu}_{o}^{\prime}\right\rangle \in \mathcal{D}_{V} \quad \boldsymbol{\nu}_{p}^{\prime}=A(\boldsymbol{\nu}, \boldsymbol{\pi}) \\
\boldsymbol{\nu} \stackrel{\langle a, \boldsymbol{\pi}\rangle}{\longrightarrow} \boldsymbol{\nu}^{\prime}
\end{gathered}
$$

The rule says that a transition $(a, \boldsymbol{p}, G, A)$ of an ioSTS is fireable in the current state $\boldsymbol{\nu}=\left\langle\boldsymbol{\nu}_{p}, \boldsymbol{\nu}_{o}\right\rangle$, if there exists a valuation $\boldsymbol{\pi}$ of the communication parameters $\boldsymbol{p}$ such that $\langle\boldsymbol{\nu}, \boldsymbol{\pi}\rangle$ satisfies the guard $G$; in such a case, the valued action $\langle a, \boldsymbol{\pi}\rangle$ is taken, the proper variables are assigned new values as specified by the assignment $A$, whereas observed variables take arbitrary values. Such a behaviour for observed variables reflects the fact that their value is defined by another ioSTS.

Given this semantics, most notions and properties of ioSTS are defined in terms of their underlying ioLTS semantics. For example, a run (resp. a trace) of an ioSTS $\mathcal{M}$ is a run (resp. a trace) of its ioLTS semantics $\llbracket \mathcal{M} \rrbracket$. An ioSTS $\mathcal{M}$ is deterministic if $\llbracket \mathcal{M} \rrbracket$ is deterministic. Whether an ioSTS is deterministic or not cannot be decided for ioSTS in the general case, as it implies the knowledge of reachable states. Sufficient conditions for an ioSTS to be deterministic exist (mutual exclusion of guards of all transitions labeled by the same action).

The product of ioSTS is more complex than that of ioLTS: ioSTS synchronize on actions, but also via observed variables, which observe runs (not only traces). 
Definition 7 (Product). Two ioSTS $\mathcal{M}^{i}=\left(V^{i}, \Theta^{i}, \Sigma, T^{i}\right), i=1,2$ with the same alphabet are compatible for product if $V_{p}^{1} \cap V_{p}^{2}=\emptyset$ (proper variables are disjoint). In this case, their product $\mathcal{M}^{1} \times \mathcal{M}^{2}=\mathcal{M}=(V, \Theta, \Sigma, T)$ is defined by

$-V=V_{p} \cup V_{o}$, with $V_{p}=V_{p}^{1} \cup V_{p}^{2}$ and $V_{o}=\left(V_{o}^{1} \cup V_{o}^{2}\right) \backslash V_{p}$;

$-\Theta\left(\left\langle\boldsymbol{v}^{1}, \boldsymbol{v}^{2}\right\rangle\right)=\Theta^{1}\left(\boldsymbol{v}^{1}\right) \wedge \Theta^{2}\left(\boldsymbol{v}^{2}\right)$;

- $T$ is defined by the following inference rule:

$$
\begin{gathered}
{\left[a(\boldsymbol{p}): G^{1}\left(\boldsymbol{v}^{1}, \boldsymbol{p}\right) ?\left(\boldsymbol{v}_{p}^{1}\right)^{\prime}:=A^{1}\left(\boldsymbol{v}^{1}, \boldsymbol{p}\right)\right] \in T^{1}} \\
{\left[a(\boldsymbol{p}): G^{2}\left(\boldsymbol{v}^{2}, \boldsymbol{p}\right) ?\left(\boldsymbol{v}_{p}^{2}\right)^{\prime}:=A^{2}\left(\boldsymbol{v}^{2}, \boldsymbol{p}\right)\right] \in T^{2}} \\
{\left[a(\boldsymbol{p}): G^{1}\left(\boldsymbol{v}^{1}, \boldsymbol{p}\right) \wedge G^{2}\left(\boldsymbol{v}^{2}, \boldsymbol{p}\right) ?\left(\boldsymbol{v}_{p}^{1}\right)^{\prime}:=A^{1}\left(\boldsymbol{v}^{1}, \boldsymbol{p}\right),\left(\boldsymbol{v}_{p}^{2}\right)^{\prime}:=A^{2}\left(\boldsymbol{v}^{2}, \boldsymbol{p}\right)\right]}
\end{gathered}
$$

If $V_{o}^{1} \cap V_{p}^{2} \neq \emptyset, G^{1}$ and $A^{1}$ may depend on proper variables of $\mathcal{M}_{2}$ (cf. Figure 3).

Let $\mathcal{M}^{1}$ and $\mathcal{M}^{2}$ be two ioSTS compatible for product, and $\mathcal{M}=\mathcal{M}^{1} \times \mathcal{M}^{2}$. Then $\operatorname{Traces}(\mathcal{M}) \subseteq \operatorname{Traces}\left(\mathcal{M}^{1}\right) \cap \operatorname{Traces}\left(\mathcal{M}^{2}\right)$. Let also $F^{i}=X^{i} \times \mathcal{D}_{V_{o}^{i}}$, where $i=1,2$ and $X^{i} \subseteq \mathcal{D}_{V_{p}^{i}}$, be sets of accepting states of ioSTS $\mathcal{M}^{i}$. By taking as set of accepting states $F=X^{1} \times X^{2} \times \mathcal{D}_{V_{o}}$ for $\mathcal{M}$, we have $\operatorname{Traces}_{F}(\mathcal{M}) \subseteq$ Traces $_{F^{1}}\left(\mathcal{M}^{1}\right) \cap$ Traces $_{F^{2}}\left(\mathcal{M}^{2}\right)$. It is not hard to see that the two trace inclusions are obtained from corresponding equalities for runs and accepting runs, which become inclusions by projection on observable actions.

The testing theory for ioLTS developed in Section 2.1 also applies to ioSTS. Specifications, test purposes and test cases are assumed to be ioSTS; moreover

- A specification is supposed to be an ioSTS $S=\left(V^{S}, \Theta^{S}, \Sigma, T^{S}\right)$ with only proper variables and no observed variable $\left(V^{S}=V_{p}^{S}\right)$;

- A test purpose for $S$ is an ioSTS $T P=\left(V^{T P}, \Theta^{T P}, \Sigma, T^{T P}\right)$ such that $V_{o}^{T P}=V_{p}^{S}$ (symbolic test purposes are allowed to observe the internal state of $S$ ). The set of accepting states is defined by the truth value of a Boolean variable Accept $\in V_{p}^{T P}$. TP should be complete except when Accept $=$ true, which means that for any action $a, \bigcup_{(a, p, G, A) \in T^{T P}} G \Leftrightarrow \neg$ Accept. This condition can be enforced syntactically by completion of $T P$. It ensures that $T P$ does not restrict the runs of $S$ before they are accepted (if ever).

- A test case is an ioSTS $T C=\left(V^{T C}, \Theta^{T C}, \Sigma, T^{T C}\right)$ with a variable Verdict $\in$ $V^{T C}$ of the enumerated type $\{$ none, fail, pass, inconc $\}$.

The set of accepted traces is defined as $\operatorname{ATraces}(S, T P)=\operatorname{Traces}_{\text {Accept }}(S \times T P)$ (as in Definition 4, except that the product is now the ioSTS product).

\section{Off-line Test Selection for ioSTS}

The aim of this section is to extend the test generation principles of ioLTS to symbolic test generation, taking into account the following difficulties:

1. Ensuring semantic transformations through operations on ioSTS;

2. Relying on approximate coreachability analysis instead of exact analysis, due to undecidability issues in the (infinite-state) symbolic case. 
Table 3. Off-line symbolic test selection algorithm

$\{1$. Product and Pass verdict $\}$

$P:=S \times T P$

$P^{\prime}=\left(V^{P} \cup\{\right.$ Verdict $\}, \Theta^{P} \wedge$ Verdict $=$ none, $\left.\Sigma, T^{P^{\prime}}\right)$ is defined by

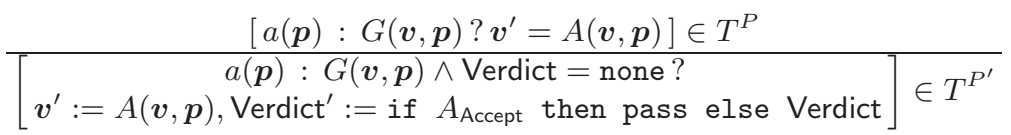

\{2. Selection and Inconc verdict \} $P^{\prime \prime}=\left(V^{P^{\prime}}, \Theta^{P^{\prime}}, \Sigma, \rightarrow^{P^{\prime \prime}}\right)$ is defined by

$$
\begin{gathered}
\frac{\left[a(\boldsymbol{p}): G(\boldsymbol{v}, \boldsymbol{p}) ? \boldsymbol{v}^{\prime}=A(\boldsymbol{v}, \boldsymbol{p})\right] \in T^{P^{\prime}}}{\left[a(\boldsymbol{p}): G(\boldsymbol{v}, \boldsymbol{p}) \wedge p r e^{\alpha}(A)\left(\text { coreach }^{\alpha}\right) ? \boldsymbol{v}^{\prime}=A(\boldsymbol{v}, \boldsymbol{p})\right] \in T^{P^{\prime \prime}}} \quad(\text { KeepI }) \\
\frac{\left[a(\boldsymbol{p}): G(\boldsymbol{v}, \boldsymbol{p}) ? \boldsymbol{v}^{\prime}=A(\boldsymbol{v}, \boldsymbol{p}) ; \text { Verdict }^{\prime}:=A \text { Verdict }\right] \in T^{P^{\prime}} \quad a \in \Sigma_{!}}{\left[a(\boldsymbol{p}): G(\boldsymbol{v}, \boldsymbol{p}) \wedge \neg \operatorname{pre}^{\alpha}(A)\left(\text { coreach }^{\alpha}\right) ? \boldsymbol{v}^{\prime}=A(\boldsymbol{v}, \boldsymbol{p}) ; \text { Verdict }^{\prime}:=\text { inconc }\right] \in T^{P^{\prime \prime}}}
\end{gathered}
$$

(Inconc)

\{3. Input-completion and Fail verdict \} $T C=\left(V^{P^{\prime}}, \Theta^{P^{\prime}}, \Sigma, T^{T C}\right)$ is defined by

$$
\frac{t \in T^{P^{\prime \prime}}}{t \in T^{T C}} \quad(\mathrm{KeepF}) \quad \frac{a \in \Sigma_{!} \quad G_{a}=\bigwedge\left\{\neg G(\boldsymbol{v}, \boldsymbol{p}) \mid(a, \boldsymbol{p}, G, A) \in T^{P^{\prime \prime}}\right\}}{\left[a(\boldsymbol{p}): G_{a}(\boldsymbol{v}, \boldsymbol{p}) ? \text { Verdict }^{\prime}:=\text { fail }\right] \in T^{T C}}
$$

We consider again the simple case where the specification $S$ and the test purpose $T P$ do not contain internal actions or non-determinism. Our running example is depicted on Figure 1-6. The selection algorithm is given in Table 3. The first step is the symbolic ven 10 n of Step 1 for ioLTS (cf. Table 2). The sar invariants hold. The transformation from $P$ to $P^{\prime}$ specifies the behavior of the Verdict variable and makes states with Verdict $\neq$ none sink.

Step 2 is the main step of the selection. As the coreachability problem is now undecidable, coreachability analysis should be approximated. Fixpoint computations on ioSTS or similar models can indeed be overapproximated by classical Abstract Interpretation techniques $[4,7,10]$. We consider here an overapproximation coreach $^{\alpha} \supseteq \operatorname{coreach}$ (Pass) $\mathrm{ft}$ the exact set of coreachable states (see Figure 7(b)). It can be represented by a logical formula to be used in syntactical operations on ioSTS. Moreover, $\operatorname{pre}^{\alpha}(A)(X)$ denotes a formula representing an overapproximation of the precondition pre $(A)(X)=A^{-1}(X)$ of states in $X$ by the assignement $A$. In this context, $\operatorname{pre}^{\alpha}(A)\left(\right.$ coreach $\left.^{\alpha}\right)$ is an overapproximation of the set of values for variables and parameters which allow to stay in coreach(Pass) when taking the transition, or in other words it is a necessary condition. Its negation is thus a sufficient condition to leave coreach(Pass), and to lose the game for the test case. Hence, rule (KeepI) discards all (semantic) transitions labeled by a (controllable) input that cerfainly exit coreach(Pass), and rule (KeepI) "redirects" to Inconc all transitions labelled by an (uncontrollable) outplet that certainly exit coreach(Pass).

Finally, Step 3 is the symbolic version of the corresponding step in Table 2. 


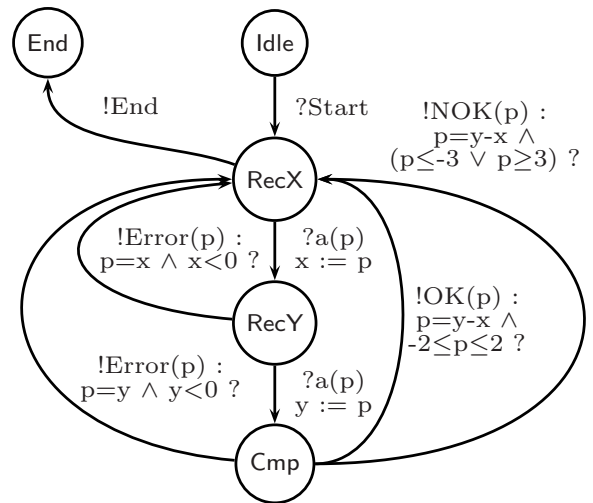

Fig. 1. Specification

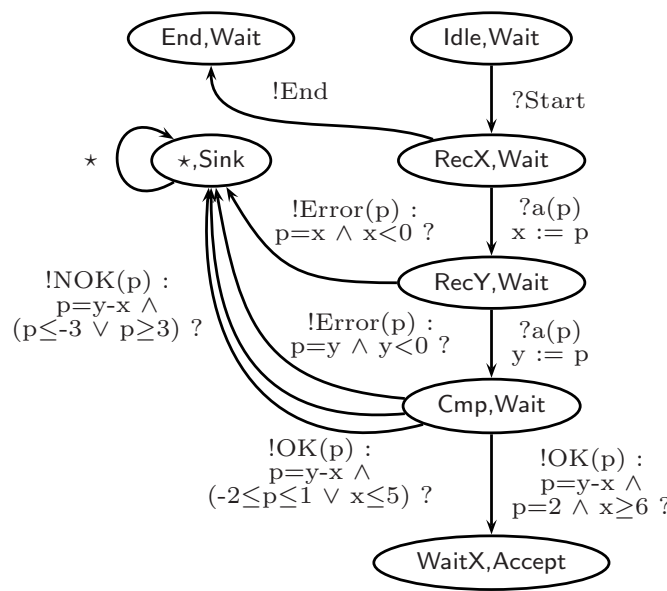

Fig. 3. Product ioSTS (after Step 1)

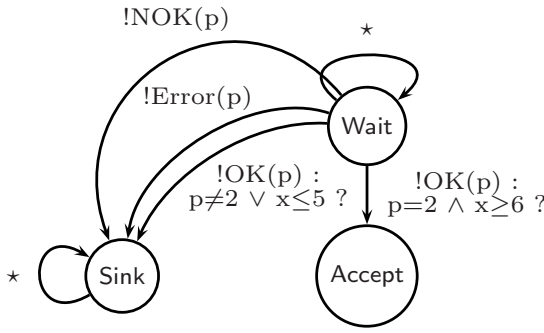

Fig. 2. Test Purpose

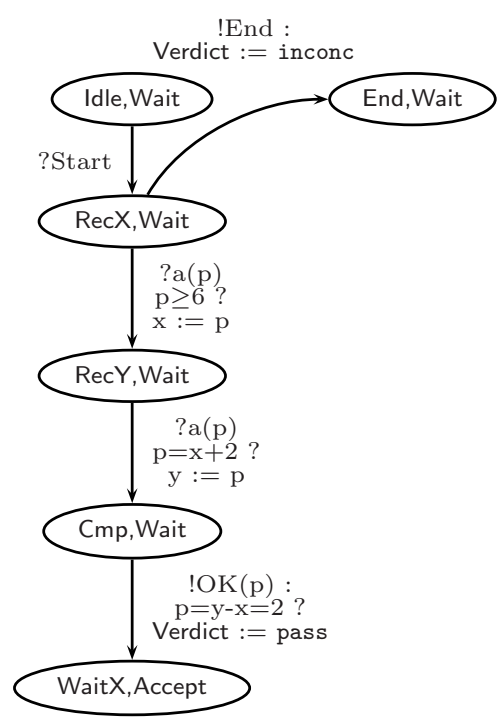

Fig. 4. Product ioSTS modified using coreachability analysis and Inconc verdict (Step 2), and simplified with reachability analysis

Example: The specification describes a program which is waiting for two successive inputs ? $a\left(p_{1}\right)$ and $? a\left(p_{2}\right)$, and emits $! O K\left(p_{2}-p_{1}\right)$ when their difference is lo s than 2 in absolute value, and !NOK otherwise. If the value held by the channel $? a$ is negative, the message !Error is emitted. The program may also emit !End and ends its execution. The test purpose specifies that a test of interest is one that terminates with the first emission of $! O K(p)$, and with $p=2$, from a state of the specification where $x \geq 6$. A !NOK $(p)$ message is forbidden. This implies that we should have $p \geq 6$ (resp. $p=x+2$ ) in $? a(p)$ from location RecX (resp. RecY), facts which are discovered by a coreachability analysis using convex polyhedra [7] and taken into account in Figure 4. The resulting test case is depicted in Figure 5.

In contrast, if the analysis performed on the product of Figure 3 a more simple interval analysis, it would only detects that we should have $p \geq 6$ in ?a $(p)$ from location RecY, and we would obtain the test case depicted in Figure 6 . Here, we avoid to lose the game by receiving a conformant ?Error $(p)$ messages (because we emit $! a(p)$ with $p \geq 0)$ but we can lose the game with conformant ?OK $(p)$ ol $\$ N O K(p)$ messages. 


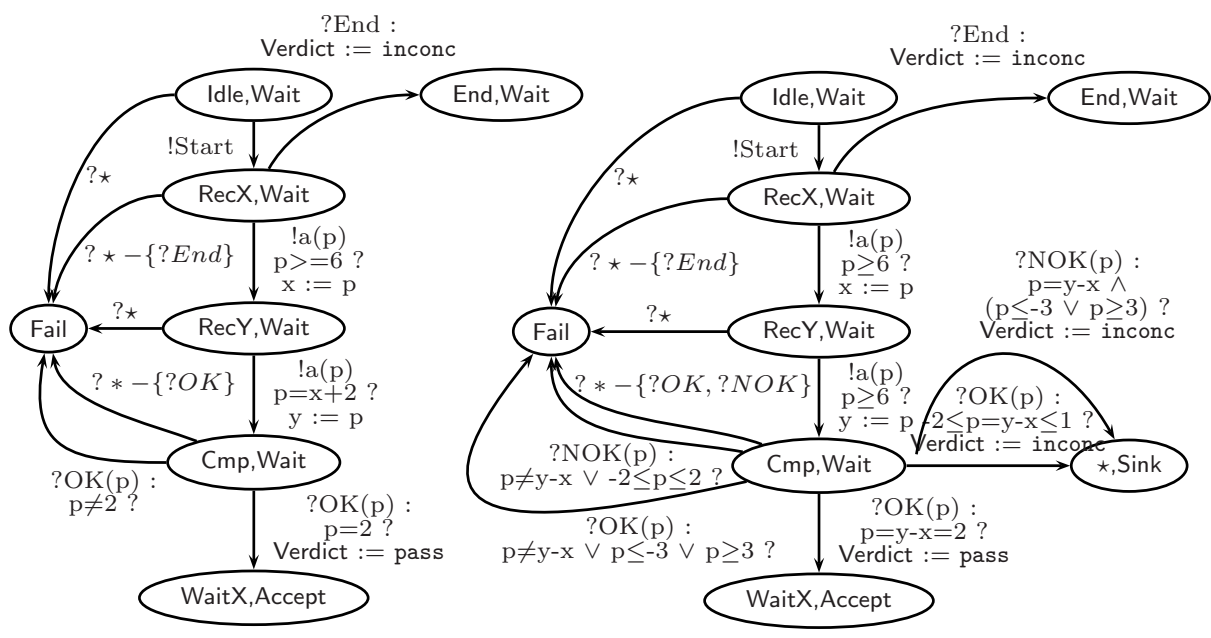

Fig. 5. Resulting Test Case

Fig. 6. Less accurate Test Case

Intuitively, the main effect of approximations for the generated test case will be either to miss the Inconc verdict, when receiving IUT outputs, or to lose the game, when sending inadequate inputs to the IUT, as illustrated by the comparison of Figure 5 and 6 In the worst case, when the approximation does not deliver any information, the Inconc verdict will never be emitted and the test case will not guide at all the parallel execution towards accepting states. This will be formalized in Section 6

\section{On-line Test Selection and Test Case Execution}

The off-line test selection phase produces an ioSTS equipped with verdicts, in which (some) losing strategies has been removed, but it does not implement a single strategy, in terms of its game against the IUT. Indeed, it may contain choices between several outputs that may be sent to the IUT. For instance, in Figure 5] from the location RecY,Wait, the action $a(p)$ may be emitted for any $p \geq 6$. This illustrates the fact that the test case has to assign values to the formal communication parameters carried by the actions.

As a consequence, test case execution implies on-line constraint solving. A test case is then executed as follows. At any point of the parallel execution of $T C$ with IUT, $T C$ is in a known state $\boldsymbol{\nu} \in \mathcal{D}_{V}$. It may then have the choice between observing an output of the IUT, or controling an input of the IUT:

- TC observes an output $a$ of the IUT with actual values of parameters $\pi \in$ $\mathcal{D}_{\text {sig(a) }}$. As $T C$ is input-complete and deterministic, exactly one transition $(a, \boldsymbol{p}, G, A) \in T^{T C}$ satisfies $\langle\boldsymbol{\nu}, \boldsymbol{\pi}\rangle \in G$. It then performs the assignments $\boldsymbol{\nu}^{\prime}=A(\boldsymbol{\nu}, \boldsymbol{\pi})$, and checks the value of Verdict.

- $T C$ controls an input $a$ in a transition $(a, \boldsymbol{p}, G, A)$. By constraint solving, it chooses $\boldsymbol{\pi}$ such that $\langle\boldsymbol{\nu}, \boldsymbol{\pi}\rangle \in G$, sends $a(\boldsymbol{\pi})$ to $I U T$, performs $\boldsymbol{\nu}^{\prime}=A(\boldsymbol{\nu}, \boldsymbol{\pi})$, and finally checks the verdict. 
A test execution driver thus needs to implement the choice between observing or controlling, the evaluation of guards on outputs of the IUT, constraint solving for the choice of values of parameters on inputs of the $I U T$, and evaluation of assignments. Evaluation of a formula is never a problem, however the use of constraint solving techniques to instantiate input parameters imposes restrictions into a decidable theory, such as Presburger arithmetic.

\section{Quality of Generated Test Cases}

As sketched during the off-line test selection algorithm of Section 4 test case verdicts are sound, which implies the usual soundness property of test cases only non-conformant IUT can be rejected, like for ioLTS. Exhaustiveness every non conformant IUT may be rejected [16], can also be proved, but this is out of the scope of the present paper.

These properties are related to conformance or to soundness of verdicts. They do not say whether test cases are good players in the game against the IUT . In this section we formalize qualitative properties of test cases relative to their ability to satisfy the test purpose during test execution, and show how the precision of the approximate analysis during the off-line selection algorithm influences them. We consider a fixed specification $S$ and test purpose TP.

We can first compare two test cases in terms of their sets of traces leading to Pass. The requirement (4) of Table 1 only relates $\operatorname{Traces}_{\text {Pass }}(T C)$ and Traces $(T C)$. However, a test case can be pruned in any state where there exists a choice between several outputs (inputs of $I U T$ ). Such an operation may reduce the sets Traces $(T C)$ and Traces Pass $(T C)$.

Definition 8 (Completeness ordering; completeness of a test case). Let $T C$ and $T C^{\prime}$ be two test cases with sound verdicts (Definition 5), both generated from same $S$ and $T P . T C^{\prime}$ is less complete than $T C$, denoted by $T C^{\prime} \preceq^{\text {comp }} T C$, if Traces $_{\text {Pass }}\left(T C^{\prime}\right) \subseteq$ Traces $_{\text {Pass }}(T C) . T C$ is a complete test case if $\operatorname{Traces}_{\text {Pass }}(T C)=\operatorname{ATraces}(S, T P)$.

The test cases produced by the off-line test selection algorithms of Sections 2.3 and 4 are complete. In the TGV tool however [9], they are pruned to remove the choices between inputs to be sent to the IUT. Thus, in this case the on-line test selection (described in section 5 for ioSTS) is partly performed off-line.

The (partial) completeness of a test case is not directly related to its quality as a player in the game for satisfying the test purpose. However, we are only able to compare the quality of test cases when they are equivalent with respect to the completeness ordering. Otherwise, two test cases may have disjoint sets of traces, which makes their comparison as players difficult. We now define an accuracy ordering between test cases that are equivalent with respect to the completeness ordering. The definition seems simplistic, however it makes sense when examining its consequences, by taking the properties of Table 1 into account.

Definition 9 (Accuracy ordering). Let $T C$ and $T C^{\prime}$ be two test cases with sound verdicts that are equivalent for the completeness ordering. TC is more accurate than $T C^{\prime}$, denoted by $T C \preceq{ }^{\text {acc }} T C^{\prime}$, if $\operatorname{Traces}(T C) \subseteq \operatorname{Traces}\left(T C^{\prime}\right)$. 


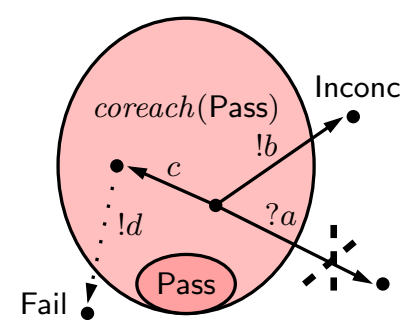

(a) Exact analysis

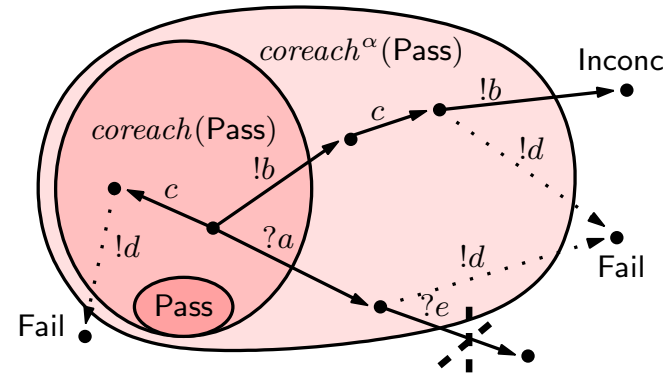

(b) Approximate analysis

Fig. 7. Control and inconclusiveness: Step 2 of off-line selection algorithms

In particular, using properties of Table 1, $T C \preceq$ acc $T C^{\prime}$ implies

1. Traces Inconc $(T C) \subseteq \operatorname{Traces}\left(T C^{\prime}\right): T C^{\prime}$ detects inconclusives later than $T C$, if ever;

2. Traces $_{\text {Fail }}(T C) \subseteq$ Traces $_{\text {Fail }}\left(T C^{\prime}\right): T C^{\prime}$ emits more Fail verdicts than $T C$,

The second consequence may seem paradoxical: an accurate test case detects less conformance errors than a less accurate one! In fact, two test cases that are equivalent for the completeness ordering have same accepted traces and detect exactly the same errors along prefixes of those accepted traces. But a less accurate test case $(i)$ exercices weaker control on the inputs of the IUT and $(i i)$ emits Inconc verdicts later, which gives more opportunity to the IUT to exhibit non-conformance. Figure 5 and 6 illustrates point $(i)$.

Now, among sound and completeness-equivalent test cases, the optimal test case can be defined as the test case where refused traces of $S$ w.r.t. TP are never entered by a controllable input of the $I U T$ and where Inconc verdict is emitted as soon as an output of $I U T$ enters these refused traces.

Definition 10 (Optimal test case). TC is optimal w.r.t. $S$ and TP if:

1. Traces $(T C) \cap \Lambda^{*} \cdot \Lambda_{\text {? }} \subseteq \operatorname{pref}_{\leq}(\operatorname{ATraces}(S, T P))$ : TC does not lose the game on a controllable action;

2. $\operatorname{Traces}_{\text {Inconc }}(T C)=\operatorname{Traces}(T C) \cap \operatorname{RTraces}(S, T P) \cap \operatorname{pref}_{<}(\operatorname{ATraces}(S, T P))$. $\Lambda_{!}: T C$ immediately detects refused traces.

These conditions correspond to properties (7) and (6) of Table 1, respectively.

In the case of finite ioLTS it is not hard to see that the algorithm in Section 2.3 builds sound, complete and optimal test cases. Completeness is obtained at Step 1 of the algorithm. Optimality is obtained at Step 2, as Inconc is reached exactly when leaving coreach(Pass). Both properties are preserved by Step 3 . In the case of ioSTS, we also build sound and complete test cases. However, the effect of the approximate coreachability analysis is to relax the optimality (see also Figure 66). The following theorem confirms the relevance of the accuracy ordering of test cases (Definition 9) and identifies the consequences of an approximate analysis in the off-line test selection algorithm. 
Theorem 1 (Relating accuracy to precision). Let $T C$ and $T C^{\prime}$ be two test cases generated by the algorithm described in Table 3 , where TC was generated using a more precise approximation $\alpha$ than the approximation $\alpha^{\prime}$ used for generating $T C^{\prime}$, i.e., $\operatorname{pre}^{\alpha}(A)\left(\right.$ coreach $\left.^{\alpha}\right) \subseteq$ pre $^{\alpha^{\prime}}(A)\left(\right.$ coreach $\left.^{\alpha^{\prime}}\right)$. Then $T C \preceq^{\text {acc }} T C^{\prime}$.

Proof. We need only to consider Step 2. For inputs, only the rule (KeepI) of Step 2 of the algorithm applies. In this case, a better precision for $\operatorname{pre}^{\alpha}(A)\left(\operatorname{coreach}^{\alpha}\right)$ strengthens the guards of the symbolic transitions, implying that fewer semantic transitions will be inferred in the underlying ioLTS. For outputs, both inference rules apply. For any state $q$ of the underlying ioLTS, a better precision for $\operatorname{pre}^{\alpha}(A)\left(\right.$ coreach $\left.^{\alpha}\right)$ means that more semantic transitions from $q$ leading to Inconc (which is sink) will be inferred by the rule (Inconcl) while less transitions from $q$ to its "normal" successors (which are generally not sink) will be inferred by the rule (KeepI). This implies that $\operatorname{Traces}\left(T C^{1}\right) \subseteq \operatorname{Traces}\left(T C^{2}\right)$. Moreover, Traces $_{\text {Pass }}\left(T C^{1}\right)=$ Traces $_{\text {Pass }}\left(T C^{2}\right)$, hence the conclusion of the theorem.

The two extreme cases are actually the following:

- The computation is exact; an optimal test case is obtained, as in Section 2.3

- The approximation is maximal $\left(\right.$ pre $^{\alpha}(A)\left(\right.$ coreach $\left.^{\alpha}\right)=$ true $)$, thus delivers no information: Step 2 of the algorithm has no effect and the test case is unable to control the implementation to satisfy the test purpose, nor to detect when Inconc should be emitted.

\section{Conclusion}

In this paper, we have presented a symbolic test generation algorithm for specifications and test purposes given as symbolic automata. Test generation has been decomposed into an off-line selection of test cases and an on-line execution on the IUT. The off-line selection is based on syntactical transformations and is parameterized by an approximate fixpoint analysis. We have showed how the precision of the analysis influences the accuracy of selected test cases. The on-line execution is based on constraint solving. These algorithms have been implemented in our tool STG, STG relies on NBac (http://www.irisa.fr/prive/bjeannet/nbac/nbac.html)for theapproximate fixpoint analysis and Omega (http://www.cs.umd.edu/projects/omega/) for constraint resolution in Presburger arithmetic. STG uses a more general model than the one presented here, in particular admitting non-deterministic specifications under some restrictions. While most other works are limited to controlable (and deterministic) systems, we are able to generate test cases for non-controllable (and non-deterministic) specifications. Moreover, we believe that our framework can be generalized to other models that cannot be analyzed in an exact way.

In a sense, our approach is an improvement of strictly on-line approaches (as e.g. TorX [2,5]), which lack control of the test cases on the IUT. Off-line symbolic selection seriously improves this feature. In fact, off-line selection based on test purposes can be seen as a syntactic slicing of the specification w.r.t. particular scenarios, preserving the capability to generate sound test cases on line. 
Several extensions of our work can be investigated. We have presented this work in the context of conformance testing, but similar techniques could be used in structural testing for the selection of test cases based on the source code. Also, other models can be considered, such as programs with recursive calls modelled as pushdown automata. One problem is then to decide what is observable and controlable by a tester. Finally, other selection criteria can benefit from our techniques, like safety properties [15] or standard structural coverage criteria.

\section{References}

1. ISO/IEC 9646. Conformance Testing Methodology and Framework, 1992.

2. A. Belinfante, J. Feenstra, R.G. de Vries, J. Tretmans, N. Goga, L. Feijs, S. Mauw, and L. Heerink. Formal test automation: A simple experiment. In $12^{\text {th }}$ Int. Workshop on Testing of Communicating Systems. Kluwer Academic Publishers, 1999.

3. M. Benjamin, D. Geist, A. Hartman, G. Mas, R. Smeets, and Y. Wolfsthal. A feasibility study in formal coverage driven test generation. In DAC99, 1999.

4. P. Cousot and R. Cousot. Abstract intrepretation: a unified lattice model for static analysis of programs by construction or approximation of fixpoints. In 4th $A C M$ Symposium on Principles of Programming Languages, Los Angeles, CA, 1977.

5. L. Frantzen, J. Tretmans, and T. Willemse. Test generation based on symbolic specifications. In FATES 2004, Linz, Austria, 2004.

6. J. Grabowski, D. Hogrefe, and R. Nahm. Test Case Generation with Test Purpose Specification by MSCs. In $6^{\text {th }}$ SDL Forum, Darmstadt (Germany). North-Holland, 1993.

7. N. Halbwachs, Y.E. Proy, and P. Roumanoff. Verification of real-time systems using linear relation analysis. Formal Methods in System Design, 11(2), 1997.

8. H.S. Hong, I. Lee, O. Sokolsky, and H. Ural. A temporal logic based theory of test coverage and generation. In TACAS '02, Grenoble, France, LNCS 2280. Springer-Verlag, 2002.

9. C. Jard and T. Jéron. TGV: theory, principles and algorithms. International Journal on Software Tools for Technology Transfer, 6, 2004.

10. B. Jeannet. Dynamic partitioning in linear relation analysis. application to the verification of reactive systems. Formal Methods in System Design, 23(1), 2003.

11. B. Jeannet, T. Jéron, V. Rusu, and E. Zinovieva. Symbolic test selection using approximate analysis. Technical Report 1649, INRIA, 2004. Available at http://www.irisa.fr/bibli/publi/pi/2004/1649/1649.html.

12. T. Jéron and P. Morel. Test generation derived from model-checking. In $C A V^{\prime} 99$, Trento, Italy, LNCS 1633. Springer-Verlag, 1999.

13. D. Lee and M. Yannakakis. Principles and Methods of Testing Finite State Machines - A Survey. Proceedings of the IEEE, 84(8), 1996.

14. V. Rusu, L. du Bousquet, and T. Jéron. An approach to symbolic test generation. In Integrated Formal Methods (IFM'00), Dagstuhl, Allemagne, LNCS 1945. Springer Verlag, 2000.

15. V. Rusu, H. Marchand, and T. Jéron. Verification and symbolic test generation for safety properties. Technical Report 5285, INRIA, 2004. Available at http://www.inria.fr/rrrt/rr-5285.html.

16. J. Tretmans. Test generation with inputs, outputs and repetitive quiescence. Software-Concepts and Tools, 17(3), 1996. 